\title{
Alternatives to sugar
}

\section{The search for an ideal non-nutritive sweetener is almost a century old. K. J. Parker outlines its history}

\begin{abstract}
SWEETNESS has long been known as a property possessed by many substances other than sugar, although their use as sweeteners has largely been confined to pharmacy, or precluded by toxicity. For example, chloroform, which was discovered in 1832 , is still used for sweetening lozenges and linctus. The intensely sweet taste of liquorice root was known to the ancient Egyptians, an extract having been found in the tomb of King Tut (1500 B.C.). Although its strong liquorice flavour and pharmacological activity today limit its wider application as a sweetener, it is used in pharmacy, in some confectionery, and as a flavour potentiator.
\end{abstract}

The commercial development of artificial sweeteners dates from the discovery of saccharin in 1879 , by $C$. Fahlberg and I. Remsen, followed by its manufacture five years later. In that year, the intense sweetness of paraethoxyphenylurea was discovered by $\mathbf{J}$. Berlinerblau, and within ten years, this compound was being marketed under the name of dulcin.

By the end of the last century sugar had become accepted as a basic food item, and its use and ready availability were taken for granted. Saccharin provided an alternative sweetener to sucrose, for use in times of sugar shortage, as a cheap substitute for carbohydrate sweeteners, or for inclusion in sugar-free diets. Its slightly bitter flavour and metallic after-taste. however, contrasting with the pure sweet taste of sucrose, reduces its acceptability. Consequently, the production of saccharin, which in Germany had reached $100,000 \mathrm{lb} /$ year by the turn of the century and increased sharply during the two world wars, remained fairly static in Britain and Europe for the first half of this century.

Before 1940, inadequate nutrition had been a major concern of world health authorities. A decade later it began to be more widely appreciated that the converse-excessive or unbalanced food intake-could be equally injurious to health, possibly contributing to heart diseases, obesity and metabolic disorders. Since the need for essential dietary factors - vitamins, minerals, amino acids-was recognised, the emphasis for control of nutritional intake inevitably focused on carbohydrates and especially on sugar. Although the polemics of this particular view are still being debated, the substitution of sugar in the diet by a non-nutritive alternative has already proved to be a painless and popular means of reducing carbohydrate-and hence, calorie-intake.

The demand for saccharin and its particular shortcomings meant that the market was ready for a more acceptable alternative. This was provided by a new sweetener, N-cyclohexylsulphamic acid, or cyclamate, discovered in 1937 by L. F. Audrieth and M. Sveda. In spite of its having only less than one tenth the sweetness of saccharin, the flavour of cyclamate was considered to be singularly pleasant and free from after-taste. Admixture of $10 \%$ saccharin effectively doubled its sweetness without introducing undesirable after-taste.

\section{Legislation on sweeteners}

The use of saccharin-cyclamate sweeteners, particularly in soft drinks, which currently accounts for some $70 \%$ of the synthetic sweeteners consumed, increased tenfold between their introduction and 1968. By then, concern was mounting over the potential hazard of unrestricted consumption of artificial sweeteners, epecially by children, as a consequence of the widespread use of saccharin-cyclamate in non-dietetic foods and beverages.

A major manufacturer, Abbott Laboratories, had spent an estimated $\$ 1$ million on establishing the safety of this sweetener. Ironically, their disclosure that massive doses of cyclamate fed to rats was associated with the development of malignant bladder tumours in some of the test animals led in October 1969 to an immediate ban on the use of cyclamates in the United States. Great Britain, Canada and several other countries followed with equivalent legislation, though the restriction was not universal. Saccharin the only remaining permitted artificial sweetener, was subsequently removed from the Food and Drug Administration (FDA) list of food additives generally recognised as safe (GRAS list). Finally, in March of this year, following the results of at least six separate studies on rats in which cancerous tumours were positively identified, the use of saccharin in diet foods, cosmetics and non-prescription drugs was ordered to be withdrawn in the United States within two years, under the controversial 1958 Delaney amendment to the 1938 Food, Drug and Cosmetic Act. The Delaney clause makes mandatory the banning of the food use of any substance shown to induce cancer in any animal under any conditions.
It was against this background of an increasingly urgent demand for a safe non-nutritive sweetener that research into the chemical basis of sweetness was boosted in the late 1960 s. In spite of the extensive research into the physiology and psychology of taste perception, however, it is still not possible to predict the chemical structure of an ideal non-nutritive sweetener, or even that a substance will be sweet. Many types of compound are known to taste sweet, but in every class the initial discovery of sweetness was accidental. There seems to be no common molecular property which can be used to predict the ability to elicit a sweet taste.

Theoretical models have been advanced for the molecular basis of sweetness, the most comprehensive being that of Robert Shallenberger of Cornell University. In its most recent form, his model accounts for the perception of sweetness in terms of a three-point interaction with the taste receptor protein, involving a proton donor, a proton acceptor and a lipophilic group sited at the points of a precisely specified triangle. In spite of the ingenious explanation of the sweetness of known compounds, however, not all compounds fitting the model are sweet and the theory cannot be used to predict or construct radically new sweeteners.

\section{Nature and nurture}

Research into new sweeteners thus takes the form of molecular variations on a known structural theme. Alternatively, intensely sweet substances of natural origin can be sought and characterised. Either approach, however, is ultimately subject to the requirement that the candidate sweetener should meet the increasingly rigorous toxicological safety standards imposed by the public health authorities, before it can be permitted for use as a food additive. Since toxicity cannot be predicted on the basis of molecular structure either, the search is inevitably speculative.

Since the ban imposed on cyclamate, Abbott Laboratories have been continuing to sponsor research into the toxicology and safety of cyclamate, and in November, 1973, petitioned the FDA for its reinstatement on the grounds of need and in the light of further experimental evidence. It is argued that the single piece of evidence indicting cyclamate was not substantiated by subsequent studies. With the 
eventual withdrawal of saccharin there will be no permitted sweetener on the US market, a situation in which the FDA may be obliged to reconsider rescinding the earlier ban on cyclamate, though so far the Abbott petition has not been allowed.

\section{New synthetic sweeteners}

Several candidate sweeteners are being developed, but very little published information is available. The market is highly competitive, and no wonder for production of a multi-billion dollar sweetener is the prize.

In addition to cyclamate, two other non-calorific sweeteners, Aspartame and $\beta$-neohesperidin dihydrochalcone, have been under consideration by the FDA, though neither has yet been declared wholly acceptable. The intense sweetness of the methyl ester of the dipeptide $\alpha$-L-aspartyl-L-phenylalanine was discovered by James Schlatter working in the G. D. Searle \& Co. laboratories and reported by R. H. Mazur, J. M. Schlatter and A. H. Goldkamp in 1969. though the compound had been first synthesised three years previously within ICI. Searle filed a patent for the use of this compound as a sweetener, while ICI patented the cyclohexyl analogue at almost the same time.

Since this discovery, several hundred closely related compounds have been synthesised, many of which have proved sweet. However, Searle decided to develop and market the original dipeptide methyl ester under the name of Aspartame, in conjunction with the Japanese Ajinomoto Company, which had independently developed a cheaper synthetic route. The possible formation of the cyclic self-condensation product diketopiperazine, formed in soft drinks on storage, for example, resulted in Searle voluntarily withholding the product from the market, even though qualified approval had been granted by the FDA in 1974 for the use of Aspartame as a table sweetener. Then in December, 1975, the FDA rescinded its earlier order indefinitely, pending further review of the evidence on safety, in spite of the removal of earlier fears on the carcinogenic properties of diketopiperazine.

In a study of the bitter principles of citrus fruit rind, R. M. Horowitz and B. Gentili discovered that the bitter flavanone naringin from grapefruit peel could easily be converted into the corresponding dihydrochalcone which by contrast was found to be intensely sweet, a property shared by a number of related dihydrochalcones. Of these $\beta$-neohesperidin dihydrochalcone was selected for toxicological and metabolic studies by the Southern Regional Laboratory of the US Department of Agriculture. The pilot plant production was also developed making possible its manufacture in ton quantities when required. Although its low solubility in water and the delay in taste perception make this sweetener unsuitable for many applications, once granted food additive status it could be used for sweetening chewing gum, toothpastes, pharmaceutical preparations, etc where such properties are not a disadvantage.

A new class of sweeteners, the oxathiazinone dioxides, was reported in 1973 by K. Clauss and H. Jensen of Hoescht. These, like saccharin and cyclamate, contain the sulphonamide group and a lipophilic part. The $\beta$ methyl derivative, which has been reported to have the best flavour profile, is being developed by Hoechst under the name Acetosulpham. It has ap-

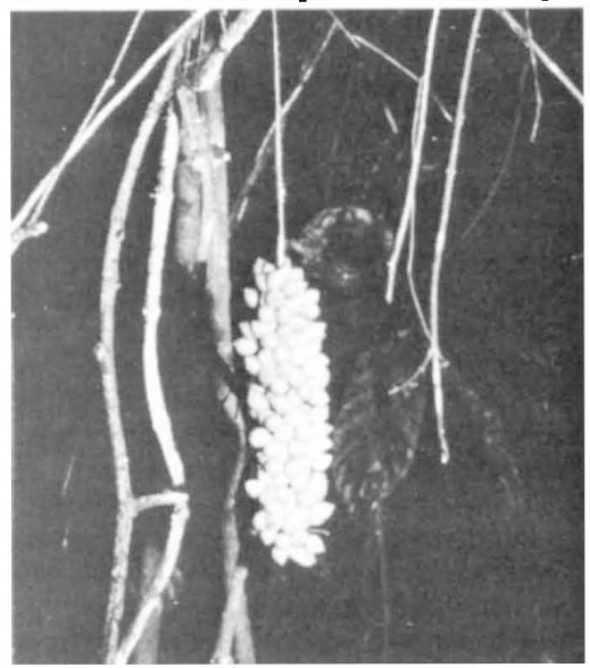

Wild serendipity berries

proximately one third the sweetness intensity of saccharin.

Although several amino acids are known to taste sweet, it was discovered accidentally, by Kornfeld of Eli Lilly and Co., that some 6- substituted Dtryptophan derivatives are intensely sweet. Of these, 6-chlorotryptophan, reported to be approximately 1,000 times as sweet as sucrose, has been selected for development as a sweetener by the company.

A systematic study of the relationship between taste and chemical structure of a series of terpene oximes and related compounds has been undertaken by a research team at Stanford University led by E. M. Acton. The intense sweetness of the $\alpha$-syn-oxime of perillartine was first reported in 1920 by Furukawa in Japan, although this compound is unsuitable for use as a sweetener owing to its low water solubility. However, a closely related oxime has been proposed as a suitable candidate sweetener by the Stanford group.

Since the early discovery of the intense sweetness of alkoxyaromatic amines--examples of which are Dulcin and, more recently, the deep yellow $\mathrm{P} 4000$, used briefly as a sweetener in
Holland during the war-numerous intensely sweet arylamines have been described. These compounds are generally toxic, and are thus unlikely to be selected for further development. However, A. Zaffaroni, of the Dynapol Corporation of America, has conceived the idea of linking a sweet molecule to a non-absorbable, inert polymer in such a way that the taste interaction of the sweet part is unaffected. Not being metabolised, the sweetener cannot manifest systemic toxicity and is non-calorific.

Why sugars and polyhydric alcohols reach a limiting level of sweetness with sucrose, while non-hydroxylic compounds of similar molecular weight can be several orders of magnitude sweeter

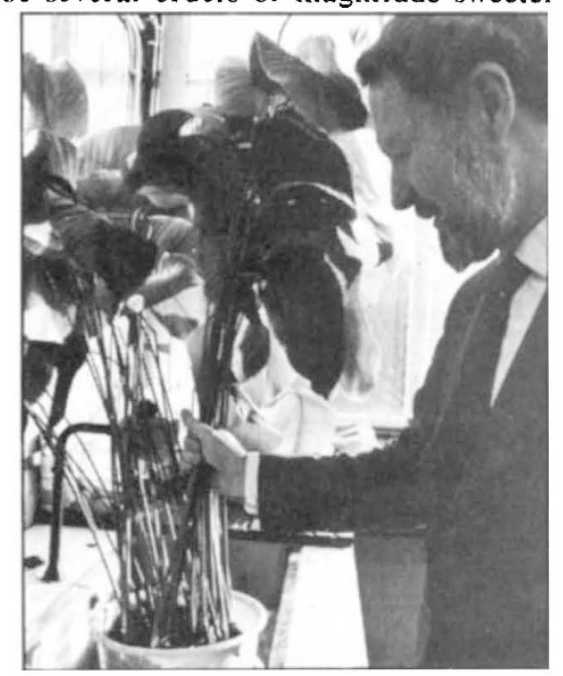

Thaumatococcus plants

can be explained by the absence of a lipophilic group from the sugar molecule. Pursuing this theme, Michael Lindley and Gordon Birch of the National College of Food Technology, Reading University, prepared several methyl ethers of sucrose but none was found to be particularly sweet. This approach was vindicated, however. with the discovery by Leslie Hough and S. P. Phadnis of Queen Elizabeth College, London, of the intense sweetness of tetrachlorogalactosucrose, while working in collaboration with Riaz Khan of Tate \& Lyle, on deoxy-and halodeoxysucrose derivatives. They subsequently showed that sweetness reaches a maximum in $1^{\prime}, 4,6^{\prime}$-trichlorotrideoxygalactosucrose, found to be approximately six hundred times sweeter than sucrose. It has a pure sweet fiavour closely similar to that of sucrose. Although it has been shown not to be toxic to rats and to be noncalorific, its suitability as a non-nutritive sweetener has yet to be established.

\section{Natural sweeteners}

The occurrence of high-intensity sweeteners in nature has long been recognised, many having been used traditionally for generations: for ex- 
ample, glycyrrhizin from the roots of the liquorice plant, the fruit of the South Chinese Lo Han Kuo and the leaves of the Paraguayan shrub Stevia rebaudiana. Stevioside, a triterpene glycoside, which is readily extracted from the leaves of $S$. rebaudiana, is being produced on a commercial scale in Japan by the Toyo Menka Karisha Company. Stevioside, which is approximately 300 times sweeter than sucrose, is not a permitted food additive elsewhere.

More recently the serendipity berry (Dioscoreophyllum cumminsii) was identified in a broad survey of sweet fruits undertaken by George Inglett and J. F. May, Department of Agriculture, in 1968. Its berries are intensely sweet owing to the presence of the protein monellin which is approximately 2,000 times as sweet as sucrose. A similar protein, thaumatin, has been isolated from the fruit of a West African plant Thaumatococcus daniellii by $H$. van der Wel and $K$. Loeve of Unilever. This has proved to be the sweetest substance known, being around 4,000 times sweeter than sucrose. The extraction, purification and properties of both monellin and thaumatin have been studied extensively, in particular by Tate \& Lyle, who are able to produce thaumatin in kilogramme quantities. However, the toxicology of thaumatin has not been fully evaluated, though, being a vegetable protein which has been consumed for generations, it seems unlikely that it would have harmful side-effects.

Similar considerations would seem to apply miraculin, the glycoprotein extracted from the berries of the bush Synsepalum dulcificum, also native to West Africa. An extract of this fruit, which has the property of tasting intensely sweet only in the presence of acids, was marketed for a time by the Miralin Corporation of America who established extensive plantations in several countries to produce the extract. However, FDA approval for this product was denied in 1974 pending toxicology testing, with the consequence that it is not now commercially available.
Naturally occurring sugars and sugar alcohols, such as fructose, xylitol and maltitol, are also being evaluated as alternative sweeteners, though since they are metabolised, they cannot be regarded as non-calorific. Xylitol, produced from birch wood, is available at ten times the price of sugar. It is finding use as a sweetener in chewing gum, though it is not a permitted sweetener in Britain and, following recent evidence of potential carcinogenic activity, may be prohibited in the US.

In spite of the intense interest worldwide in developing alternative sweeteners, the ideal non-nutritive sweetener -water soluble, chemically and thermally stable, of pure flavour, nontoxic, and of high sweetness intensityhas not yet been discovered and may still be some years off. In the meantime a less satisfactory compromise seems incvitable.

K. J. Parker is General Manager, Research, with Tate \& Lyle Limited.

\section{No soft options in Carter's energy research policy}

ENERGY policy was a major plank in Jimmy Carter's campaign platform during the 1976 Presidential election. In a year when the rising cost of imported oil was causing increasing economic concern, and the late E. F. Schumacher was telling capacity audiences that "Small is beautiful", Carter promised that, if elected, he would shift the emphasis of US energy technology away from a prime dependence on nuclear fuels and towards non-nuclear alternatives, in particular renewable resources such as solar power.

But like many other campaign promises--for example that to reduce the budget of the Defense Department, now scheduled for a 9.4 per cent increase to $\$ 117.8$ billion in fiscal year 1979-Carter's energy plans have, in the face of established institutional positions and interests, undergone considerable revision in his first year of office.

The President has maintained his firm stand on the dangers of proliferation resulting from the production of plutonium by fast breeder reactors, in particular demanding the closure of the Clinch River liquid metal fast breeder reactor at Oak Ridge, Tennessee.

But the administration is still pursuing a vigorous nuclear policy through other options, including possibly a major design project for a thorium- cycle reactor. And already last year, following, in particular, moves to speed up the licensing of new reactors, Carter's earlier claims that he would turn to nuclear energy only as "a last resort" were beginning to wear thin.

Further confirmation that energy policy has changed little comes from analysis of the Department of Energy's proposed research and development budget for the fiscal year 1979. In presenting this to Congress two weeks ago, President Carter has suggested that the overall level of energy $R \& D$ remain roughly static at $\$ 2.7$ billion.

The budget contains considerable increases in commitments to research on synthetic fuels (another area in which campaign promises seem to have been forgotten). In contrast, resources for research into renewable energy sources show little change. It is proposed, for example, to raise the budget for windpower research from $\$ 37$ million to $\$ 41$ million; and solar power research, which has grown from $\$ 4$ million in 1974 to $\$ 193$ million in 1977 and \$303 million last year, will increase only to $\$ 309$ million. The increases in these two topics scarcely keep up with the expected increase in the cost of living.

There are, admittedly, some important shifts in philosophy reflected in the budget proposals. Perhaps the

\section{Sorry, for copyright reasons some images on this page may not be available online}

The inauguration of the US

National Aeronautics and Space Administration's windmill in New Mexico 